\title{
Micropolar fluid flow modelling using the boundary element method
}

\author{
M. Zadravec, M. Hriberšek \& L. Škerget \\ University of Maribor, Faculty of Mechanical Engineering, Slovenia
}

\begin{abstract}
Flows in nature are very complex and express different behaviour under different conditions. Therefore we are interested in using proper numerical models to describe the physical behaviour of such fluid flows. The micropolar fluid flow theory enables accurate computation of flows in a scale, where questions arise on the accuracy of the Navier-Stokes equation. In the present paper, the micropolar fluid flow theory is incorporated into the framework of velocity-vorticity formulation of Navier-Stokes equations. Governing equations are derived in differential as well as integral form, resulting from the application of boundary element method (BEM).
\end{abstract}

Keywords: micropolar fluid, boundary element method, numerical modelling.

\section{Introduction}

Micropolar fluid theory was developed by Eringen [2] forty years ago and has gain attention of researchers in recent years. Lukaszewicz [7] presented in his book mathematical aspects of the theory of micropolar fluids. Many of researchers worked on natural convection of micropolar fluid in rectangular enclosure (Eringen [2], Hsu et al [5], Hsu and Wang [6]). In this work a parametric study of the effect of microstructure on the flow and heat transfer in comparison with Newtonian fluid is undertaken. The results show that dependence of the microrotation term and heat transfer on microstructure parameters is significant.

In the last few years there has been significant progress on micromachining technology. Scientists argue that flows on the microscale are different from that on macroscale, described by classical Navier-Stokes equations. Papautsky et al [9] described microchannel fluid behaviour with a numerical model based on 
micropolar fluid theory and experimentally verified the model. Results showed that micropolar fluid theory present better agreement with experiment than use of classical Navier-Stokes theory. Applicability of the theory of the micropolar fluids in microchannels depends on the geometrical dimension of the flow field (Pietal [8]).

The micropolar fluid model describes the flow of fluids where the flow behaviour of microstructures affects entire flow. This model is derived from the Navier-Stokes model and takes into account rotation of particles (molecules) independently of the fluid flow and its local vorticity field. Some examples of fluids with microstructures are animal blood carrying deformable particles (platelets), clouds with smoke, suspensions, slurries and liquid crystals.

Among different approximation methods for solving problems of fluid flow BEM is increasingly gaining attention. Here, we will focus on the development of BEM for velocity-vorticity formulation of Navier-Stokes equations Škerget et al $[10,11]$, Hriberšek et al [12], and show how to incorporate the micropolar fluid theory into the BEM framework.

\section{Mathematical formulation}

Conservation laws, which define the micropolar flow, are: conservation of mass, eqn. (1), conservation of momentum, eqn. (2), conservation of microinertia eqn. (3) and conservation of energy (4):

$$
\begin{gathered}
\frac{\partial \rho}{\partial t}+\nabla \cdot(\rho \vec{v})=0 \\
\rho \frac{D \vec{v}}{D t}=-\vec{\nabla} p+\left(\lambda+2 \mu+k_{V}\right) \Delta \vec{v}-\left(\mu+k_{V}\right) \nabla \times \nabla \times \vec{v}+k_{V} \vec{\nabla} \times \vec{N}+\rho \vec{f} \\
\rho j \frac{D \vec{N}}{D t}=(\alpha+\beta+\gamma) \Delta \vec{N}-\gamma \nabla \times \nabla \times \vec{N}-2 k_{V} \vec{N}+k_{V} \vec{\nabla} \times \vec{v}+\rho \vec{s} \\
\frac{D T}{D t}=a \Delta T
\end{gathered}
$$

where is $\rho$ density, $\vec{v}$ velocity vector, $\vec{N}$ microrotation vector, $j$ microinertia, $P$ modified pressure $(P=p-\rho \vec{g} \cdot \vec{r}), \quad p$ thermodynamic pressure, $\vec{g}$ gravity aceleration vector, $\mu$ dynamic viscosity, $\lambda$ second order viscosity coefficient, $k_{V}$ vortex viscosity coefficient, $\alpha, \beta$ and $\gamma$ spin gradient viscosity coefficients, $\vec{f}$ body force per unit mass, $\vec{s}$ body torque per unit mass, $a$ diffusivity coefficient and $D(\cdot) / D t$ material derivative. If assuming that $k_{V}=\alpha=\beta=\gamma=0$ and vanishing $\vec{f}$ and $\vec{s}$, microrotation $\vec{N}$ becomes zero and the eqn. (2) reduces to the classical Navier-Stokes equation. 
If we assume that fluid is incompressible with constant material properties we can rewrite eqn. (1)-(4) in the following form for planar flow:

$$
\begin{gathered}
\vec{\nabla} \cdot \vec{v}=0 \\
\rho \frac{D \vec{v}}{D t}=-\vec{\nabla} P+\left(\mu+k_{V}\right) \Delta \vec{v}+k_{V} \vec{\nabla} \times \vec{N}+\rho F_{B} \vec{g} \\
\rho j \frac{D \vec{N}}{D t}=\gamma \Delta \vec{N}+k_{V} \vec{\nabla} \times \vec{v}-2 k_{V} \vec{N} \\
\frac{D T}{D t}=a \Delta T
\end{gathered}
$$

In momentum equation (eqn. (6)) buoyancy effect is modelled by the Boussinesq approximation included in body force $\vec{f}=F_{B} \vec{g}$. Function $F_{B}$ can be formulated by equation $F_{B}=-\beta_{T}\left(T-T_{o}\right)$, where $\beta_{T}$ is thermal volume expansion coefficient.

By taking a curl of eqn. (6) we eliminate pressure term and use velocityvorticity formulation instead of eqn. (5) and (6) with suppose that the vorticity vector representing curl of the velocity field:

$$
\begin{gathered}
\nabla^{2} \vec{v}+\nabla \times \omega=0 \\
\frac{\partial \omega}{\partial t}+\frac{\partial v_{j} \omega}{\partial x_{j}}=\left(\frac{\mu+k_{V}}{\rho}\right) \Delta \omega-\frac{k_{V}}{\rho} \Delta N+\vec{\nabla} \times\left(F_{B} \vec{g}\right) \\
\frac{\partial N}{\partial t}+\frac{\partial v_{j} N}{\partial x_{j}}=\frac{\gamma}{\rho j} \Delta N+\frac{k_{V}}{\rho j} \omega-\frac{2 k_{V}}{\rho j} N \\
\frac{\partial T}{\partial t}+\frac{\partial v_{j} T}{\partial x_{j}}=\frac{\partial T}{\partial t}+(v \cdot \nabla) T=a \Delta T
\end{gathered}
$$

where we assume that $\vec{v}, \vec{\omega}, \vec{N}$ are solenoidal vectors. Eqn. (9) is elliptic differential equation and represents the kinematics of fluid motion, expressing the compatibility and restriction condition between velocity and vorticity field functions. The kinetic part is governed by parabolic diffusion-convection vorticity transport equation eqn. (10) in which the second term on the right-hand side is an additional term to classical vorticity equation and represents connection between vorticity and microrotation flow field. Eqn. (10) is written for planar flows in which twisting and stretching term is identical to zero $(\vec{\omega} \cdot \vec{\nabla}) \vec{v}=0$. In microrotation conservation equation eqn. (11) appears microinertia $j$ which is defined as the length scale: 


$$
j=L^{2}
$$

Spin gradient viscosity coefficient $\gamma$ is proposed by Ahmadi [1] in form

$$
\gamma=\left(\mu+\frac{k_{V}}{2}\right) j
$$

\subsection{Integral representations}

Advantage of boundary domain integral method originates from the application of Green's fundamental solutions as particular weighting functions. Different conservation models can be written in the form with an appropriate selection of a linear differential operator $L[\cdot]$ in the following general form

$$
L[u]+b=0
$$

where the operator $L[\cdot]$ can be either elliptic or parabolic, $u\left(r_{j}, t\right)$ is an arbitrary field function, and the nonhomogenous term $b\left(r_{j}, t\right)$ is applied for non-linear transport effects or pseudo body forces.

\subsubsection{Integral representation for flow kinematics}

Procedure for integral representation for flow kinematics, presented with elliptic Poisson partial differential equation eqn. (9) is given in Škerget et al [10]

$$
c(\xi) v_{i}(\xi)+\int_{\Gamma} v_{i} \frac{\partial u^{*}}{\partial n} d \Gamma=e_{i j} \int_{\Gamma} v_{j} \frac{\partial u^{*}}{\partial n_{t}} d \Gamma-e_{i j} \int_{\Omega} \omega \frac{\partial u^{*}}{\partial x_{j}} d \Omega
$$

\subsubsection{Integral representation for flow kinetics}

To apply integral representation for flow vorticity, microrotation and energy eqn. (10-12), the non-homogenous velocity field $\vec{v}(r)$ must be decomposed into the constant $\vec{v}_{o}$ and a variable or perturbated part $\widetilde{\vec{v}}(r)$, so that the diffusionconvective equation with first order chemical reaction has the form

$$
\frac{\partial u}{\partial t}+\frac{\partial v_{o j} u}{\partial x_{j}}=a_{o} \frac{\partial^{2} u}{\partial x_{j} \partial x_{j}}-\beta u-\frac{\partial \tilde{v}_{j} u}{\partial x_{j}}+\frac{I_{0}}{c_{o}}=0
$$

where $a_{o}$ and $c_{o}$ are constant transport material properties, $\beta$ is the reactor rate constant, while $I_{o}$ stands for known source term. This equation can be stated as

$$
L[u]+b=a_{o} \frac{\partial^{2} u}{\partial x_{j} \partial x_{j}}-v_{o j} \frac{\partial u}{\partial x_{j}}-\beta u+b=0
$$

where $L[\cdot]$ is linear differential operator, and $b$ stands for pseudo body force term. If we suppose that we know the fundamental solution $u^{*}(\xi, s)$ satisfying 


$$
L^{*}\left[u^{*}\right]+\delta(\xi, s)=0
$$

where $L^{*}[\cdot]$ denotes the adjoint operator to $L[\cdot]$. By applying Green's theorem s for scalar field function, the following boundary-domain integral representation can be formulated as

$$
c(\xi) u(\xi)+a_{o} \int_{\Gamma} u \frac{\partial u^{*}}{\partial n} d \Gamma=a_{o} \int_{\Gamma} \frac{\partial u}{\partial n} u^{*} d \Gamma-\int_{\Gamma} u v_{o n} u^{*} d \Gamma+\int_{\Omega} b u^{*} d \Omega
$$

The pseudo body source term $b$ includes the convection for the perturbated velocity field only, source term and initial condition

$$
b=-\frac{\partial \widetilde{v}_{j} u}{\partial x_{j}}+\frac{I_{o}}{c_{o}}+\frac{u_{F-1}}{\Delta t}
$$

Rendering the eqn. (18) by applying Gauss theorem to the domain integral of pseudo body source term we can write

$$
\begin{aligned}
c(\xi) u(\xi)+a_{o} \int_{\Gamma} u \frac{\partial u^{*}}{\partial n} d \Gamma= & a_{o} \int_{\Gamma} \frac{\partial u}{\partial n} u^{*} d \Gamma-\int_{\Gamma} u v_{n} u^{*} d \Gamma+\int_{\Omega} \widetilde{v}_{j} u \frac{\partial u^{*}}{\partial x_{j}} d \Omega \\
& +\frac{1}{c_{o}} \int_{\Omega} I_{o} u^{*} d \Omega+\frac{1}{\Delta t} \int_{\Omega} u_{F-1} u^{*} d \Omega
\end{aligned}
$$

The fundamental solution of the diffusion-convective equation with first order reaction term is represented as

$$
a_{o} \frac{\partial^{2} u^{*}(\xi, s)}{\partial x_{j}(s) \partial x_{j}(s)}+v_{o j} \frac{\partial u^{*}(\xi, s)}{\partial x_{j}(s)}-\beta u^{*}(\xi, s)+\delta(\xi, s)=0
$$

Parameter $\beta$ is defined as sum time increment parameter and $\chi$ which accounts other reaction terms

$$
\beta=\frac{1}{\Delta t}+\chi
$$

The fundamental solution $u^{*}$ and his normal derivative are expressed as Škerget et al [11]

$$
\begin{gathered}
u^{*}=a_{o} \frac{1}{2 \pi a_{o}} K_{o}(\varsigma r) \exp \left(\frac{v_{o j} r_{j}}{2 a_{o}}\right) \\
\frac{\partial u^{*}}{\partial x_{j}} n_{j}=\frac{n_{j}}{2 \pi r^{2} a_{o}}\left[\varsigma r K_{1}(\varsigma r) r_{j}-\frac{r^{2}}{2 a_{o}} K_{o}(\varsigma r) v_{o j}\right] \exp \left(\frac{v_{o j} r_{j}}{2 a_{o}}\right)
\end{gathered}
$$


where the parameter $\varsigma$ is defined as

$$
\varsigma^{2}=\left(\frac{v_{o}}{2 a_{o}}\right)^{2}+\beta
$$

$K_{o}$ and $K_{I}$ are the modified Bessel function of the second kind, $r_{j}(\xi, s)$ is the vector from the source point $\xi$ to the reference point $s$, while $r$ is its magnitude $r=\left|r_{j}\right|$ and $v_{o}^{2}=v_{o j} v_{o j}$.

Table 1: $\quad$ Parameters for different conservation equations.

\begin{tabular}{|c|c|c|c|c|c|}
\cline { 2 - 5 } \multicolumn{1}{c|}{} & $\boldsymbol{u}$ & $\boldsymbol{a}_{\boldsymbol{o}}$ & $\boldsymbol{c}_{\boldsymbol{o}}$ & $\chi$ & $\boldsymbol{I}_{\boldsymbol{o}}$ \\
\hline Vorticity & $\omega$ & $\left(\frac{\mu+k_{V}}{\rho}\right)$ & 1 & 0 & $-\frac{k_{V}}{\rho} \Delta N+\vec{\nabla} \times\left(F_{B} \vec{g}\right)$ \\
\hline Microrotation & $N$ & $\left(\frac{\gamma}{\rho j}\right)$ & 1 & $\frac{2 k_{V}}{\rho j}$ & $\frac{k_{V}}{\rho j} \omega$ \\
\hline Energy & $T$ & $a$ & 0 & 0 & 0 \\
\hline
\end{tabular}

Parameters given in Table 1, together with eqn. (22) and eqn (23), give integral forms for flow vorticity eqn. (28), microrotation eqn. (29) and energy eqn. (30).

$$
\begin{gathered}
c(\xi) \omega(\xi)+\left(\frac{\mu+k_{V}}{\rho}\right) \int_{\Gamma} \omega \frac{\partial u^{*}}{\partial n} d \Gamma=\left(\frac{\mu+k_{V}}{\rho}\right) \int_{\Gamma} \frac{\partial \omega}{\partial n} u^{*} d \Gamma-\int_{\Gamma} \omega v_{n} u^{*} d \Gamma+ \\
+\int_{\Omega} \omega \widetilde{v}_{j} \frac{\partial u^{*}}{\partial x_{j}} d \Omega-\left(\frac{k_{V}}{\rho}\right) \int_{\Gamma} u^{*} \frac{\partial N}{\partial n} d \Gamma+\left(\frac{k_{V}}{\rho}\right) \int_{\Omega} \frac{\partial u^{*}}{\partial x_{j}} \frac{\partial N}{\partial x_{j}} d \Omega+ \\
+e_{i j} \int_{\Gamma} n_{i} g_{j} F_{B} u^{*} d \Gamma-e_{i j} \int_{\Omega} g_{j} F_{B} \frac{\partial u^{*}}{\partial x_{i}} d \Omega+\frac{1}{\Delta t} \int_{\Omega} \omega_{F-1} u^{*} d \Omega \\
c(\xi) N(\xi)+\left(\frac{\gamma}{\rho j}\right) \int_{\Gamma} N \frac{\partial u^{*}}{\partial n} d \Gamma=\left(\frac{\gamma}{\rho j}\right) \int_{\Gamma} \frac{\partial N}{\partial n} u^{*} d \Gamma-\int_{\Gamma} N v_{n} u^{*} d \Gamma+ \\
+\int_{\Omega} \widetilde{v}_{j} N \frac{\partial u^{*}}{\partial x_{j}} d \Omega+\left(\frac{k_{V}}{\rho j}\right) \int_{\Omega} \omega u^{*} d \Omega+\frac{1}{\Delta t} \int_{\Omega} N_{F-1} u^{*} d \Omega \\
c(\xi) T(\xi)+a \int_{\Gamma} T \frac{\partial u^{*}}{\partial n} d \Gamma=a \int_{\Gamma} \frac{\partial T}{\partial n} u^{*} d \Gamma-\int_{\Gamma} T v_{n} u^{*} d \Gamma+\int_{\Omega} T v_{j} \frac{\partial u^{*}}{\partial x_{j}} d \Omega+ \\
+\frac{1}{\Delta t} \int_{\Omega} T_{F-1} u^{*} d \Omega
\end{gathered}
$$




\subsection{Numerical algorithm}

The derived integral equations contain several new terms, composed with the classical approach (Škerget et al [10], Hriberšek and Škerget [12]) and which can therefore be easily included into the existing numerical scheme. This scheme is presented in short in figure 1.

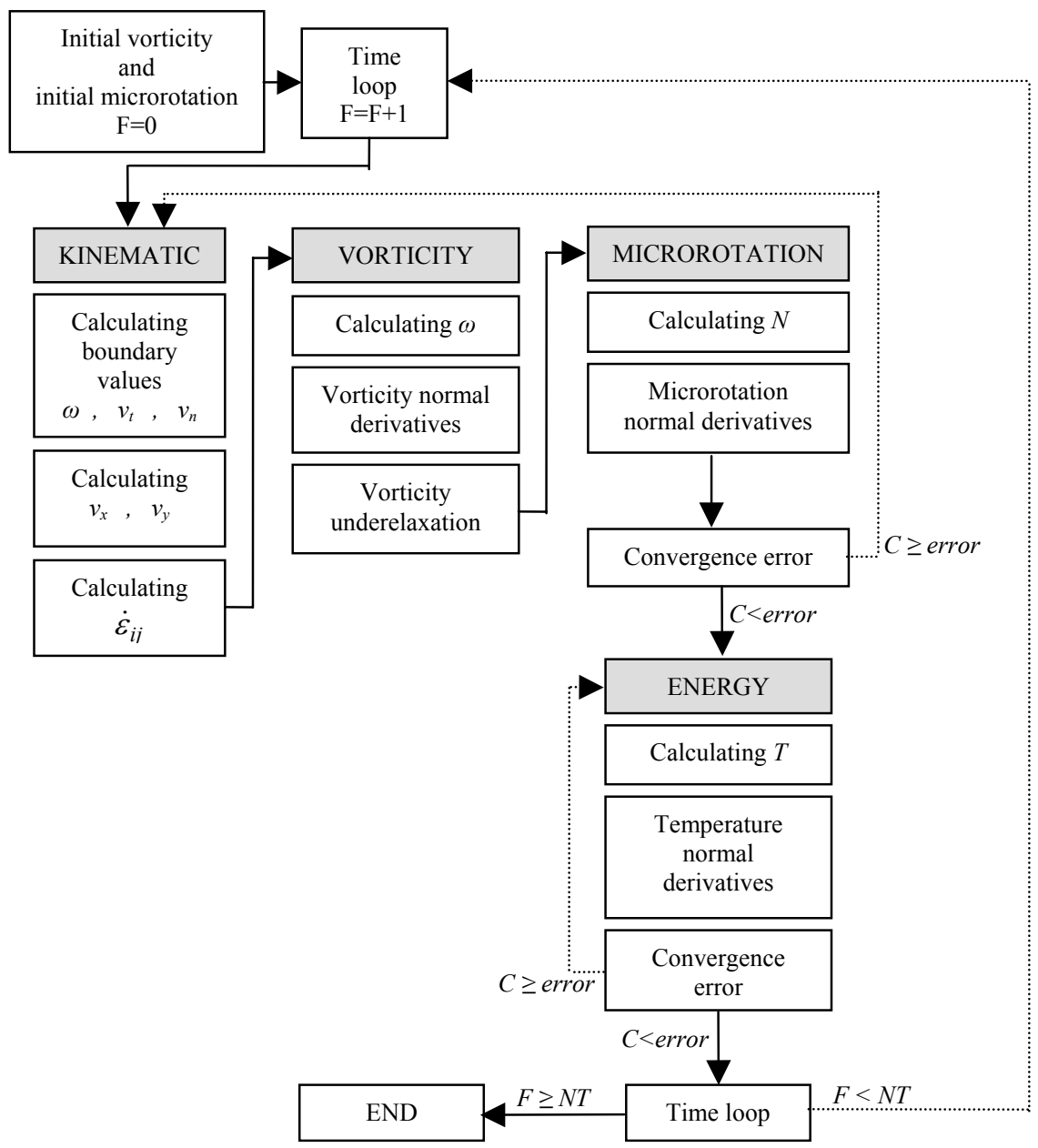

Figure 1: Flowchart scheme of micropolar theory extended BEM.

\section{Conclusions}

The paper presented the derivation of integral equations for numerical simulation of fluid flow with micropolar fluid theory. The derivation showed that the derived equations include several additional terms, compared with the set of 
equations from Navier-Stokes equations. The model will be incorporated into the BEM numerical code and tested on several benchmark problems, including flow in microchanels.

\section{References}

[1] Ahmadi, G., Self-similar solution of incompressible micropolar boundary layer flow over a semi-infinite flat plate. Int. J. Engng. Sci., 14, pp. 6396461976.

[2] Eringen, A.C., Theory of Micropolar Fluids. J. Math. Mech., 16, pp. 1-18, 1966.

[3] Eringen, A.C., Microcontinuum Field Theories. II. Fluent media, Springer Verlag, New York, 2001.

[4] Hsu, T.H. \& Chen, C.K., Natural convection of micropolar fluids in rectangular enclosure. Int.J.Engng.Sci., 34 (4), pp. 407-415, 1996.

[5] Hsu, T.H., Hsu, P.T. \& Tsai, S.Y., Natural convection of micropolar fluids in an enclosure with heat sources. Int. J. Heat and Mass Transfer, 40 (17), pp. 4239-4249, 1997.

[6] Hsu, T.H. \& Wang, S.G., Mixed convection of micropolar fluids in a cavity. Int. J. Heat and Mass Transfer, 43 (9), pp. 1563-1572, 2000.

[7] Lukaszewicz, G., Micropolar Fluids: Theory and Application, Birkhäuser, Boston, 1999.

[8] Pietal, A.K., Microchannels flow modelling with the micropolar fluid theory. Bulletin of the Polish Academy of Sciences, 52 (3), pp. 209-214, 2004.

[9] Papautsky, I., Brazzle, J., Ameel, T. \& Frazier, A.B., Laminar fluid behaviour in microchannels using micropolar fluid theory. Sensors and actuators, 73 (1-2), pp. 101-108, 1999.

[10] Škerget, L., Hriberšek, M., \& Žunič, Z., Natural convection flows in complex cavities by BEM. Int. J. Numer. Methods Heat Fluid Flow, 13 (5/6), pp. 720-736, 2003.

[11] Škerget, L., Hriberšek, M., \& Kuhn, G., Computational fluid dynamics by boundary-domain integral method. Int. J. Numer. Met. Engng., 46, pp. 1291-1311, 1999.

[12] Hriberšek, M., \& Škerget, L., Boundary domain integral method for high Reynolds viscous fluid flows in complex planar geometries. Comput. Methods Appl. Mech. Engrg., 194, pp. 4196-4220, 2005. 